\section{Juvenile idiopathische Arthritis: Score eruiert Methotrexat-Responder}

Um die Gelenkzerstörung durch eine juvenile idiopathische Arthritis (JIA) zu verhindern, müssen Methotrexat(MTX)-Nonresponder frühzeitig mit Biologika behandelt werden. Ein neuer Risikoscore hilft, solche Patienten schon vor dem Therapieversagen zu erkennen.

$\mathrm{D}$ ie Zusatztherapie mit Biologika sollte sich jedoch auf solche JIA-Patienten beschränken, die nicht oder ungenügend auf MTX ansprechen. Niederländische Ärzte haben nun einen Risikoscore entwickelt, mit dem sich besser vorhersagen lässt, ob ein Patient zu den potenziellen MTX-Respondern zählt.

Der Score beruht auf einer Kohorte von 183 therapienaiven JIA-Patienten. Von diesen wurden nach einem Jahr MTXTherapie 78,1\% gemäß ACR70-Kriterien als Nonresponder eingestuft. Bei $72 \%$ der Gesamtgruppe konnten die Ärzte die MTX-Wirkung korrekt vorhersagen, wenn sie folgende fünf Parameter berücksichtigten: die Blutsenkungsgeschwindigkeit (BSG) sowie Nukleotid-Polymorphismen (Single Nucleotide Polymorphisms, SNP) in vier Genen, die bei der Verstoffwechselung von MTX eine Rolle spielen. Anders als in früheren Studien waren Krankheitsdauer, ANA-Status und JIASubtyp in dieser Studie nicht signifikant mit dem MTX-Ansprechen assoziiert.

Aus den fünf relevanten Parametern leiteten die Mediziner einen Risikoscore für das Nichtansprechen auf MTX ab (Tab. 1). Der Score reicht von 0-11 Punkten, wobei eine höhere Punktzahl die
Gefahr des Therapieversagens erhöht. Um eine möglichst hohe Sensitivität für Nonresponse und gleichzeitig eine akzeptable Spezifität zu erreichen, legten die Autoren einen Grenzwert von $\geq 3$ Punkten fest. Damit konnten in der Ausgangskohorte $78 \%$ der MTX-Nonresponder und $49 \%$ der Responder identifiziert werden. Zur Validierung wurde der Score auf eine zweite Kohorte von 104 JIA-Patienten angewendet. Hier konnten $79 \%$ der Nonresponder (48/61) und $26 \%$ der Responder (9/34) richtig vorhergesagt werden.

Fazit: Niederländische Ärzte haben einen Risikoscore entwickelt, mit dem das Nichtansprechen von JIA-Patienten auf MTX frühzeitig aufgedeckt werden kann. Der Score beruht auf einem klinischen (BSG) und vier genetischen Faktoren (SNP) und soll jetzt hinsichtlich seiner Aussagekraft und seines klinischen Nutzens bei einer größeren Zahl von Patienten überprüft werden.

Dr. Beate Schumacher

Bulatović M et al. Prediction of clinical non-response to methotrexate treatment in juvenile idiopathic arthritis. Ann Rheum Dis; online first, published on April 3, 2012 as 10.1136/annrheumdis-2011-200942

\begin{tabular}{|c|c|c|}
\hline \multicolumn{3}{|c|}{ Vorhersage von ACR70-Nonresponse unter Methotrexat } \\
\hline \multicolumn{2}{|l|}{ Parameter } & \multirow{2}{*}{$\begin{array}{l}\text { Punktwert } \\
-2\end{array}$} \\
\hline BSG & $>12 \mathrm{~mm} / \mathrm{h}$ & \\
\hline MTRR rs1801394 A>G & $\mathrm{AG} / \mathrm{GG}^{*}$ & -3 \\
\hline MDR-1/ABCB1 rs1045642 G>A & $A A^{*}$ & -2 \\
\hline MRP-1/ABCC1 rs35592 T>C & $\mathrm{TC} / \mathrm{CC}^{*}$ & -2 \\
\hline PCFT rs2239907 C>T & $\mathrm{CT} / \mathrm{TT}^{*}$ & -2 \\
\hline \multicolumn{3}{|c|}{ *vorliegender Polymorphismus (SNP), ermittelt per PCR } \\
\hline \multicolumn{3}{|c|}{$\begin{array}{l}\text { MTRR: Methionin Synthase Reductase; MDR:Multidrug Resistance; MRP: Multidrug Resistance Protein; PCFT: Proton-cou- } \\
\text { pled Folate Transporter }\end{array}$} \\
\hline \multicolumn{3}{|c|}{ Modifiziert nach Bulatović, Ann Rheum Dis 2012.} \\
\hline
\end{tabular}

Zur Berechnung des individuellen Risikos werden die Punktwerte der zutreffenden Risiken zum Ausgangswert von 11 Punkten addiert.
Springer $\underline{\text { Springer }}$ Springer Springer

Q Springer Springer Q Springer 2 Springer Springer $\underline{\text { Springer }}$ 을 Springer Springer Springer $\underline{\text { Springer }}$ 은 Springer Springer 을 Springer Springer 은 Springer Springer Springer Springer 을 Springer Springer Q Springer $\underline{\text { Springer }}$ 을 Springer 을 Q Springer $\underline{\underline{Q} \text { Springer }}$ 을 Springer 을 Springer 은 Springer Springer Springer $\underline{\text { Springer }}$ 은 Springer Springer

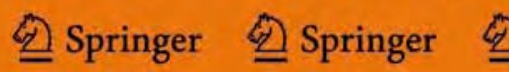
을 Springer 2 singer 을 Springer Springer 은 Springer Springer Springer Springer 을 Springer Springer

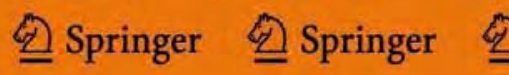
을 Springer Springer Q Springer 2 Springer 